\title{
The behavior of larval zebrafish reveals stressor-mediated anorexia during early vertebrate development
}

\author{
Rodrigo J. De Marco ${ }^{1}$, Antonia H. Groneberg ${ }^{1}$, Chen-Min Yeh ${ }^{1}$, Mario Treviño ${ }^{2}$ and Soojin Ryu ${ }^{1}$ * \\ 1 Developmental Genetics of the Nervous System, Max Planck Institute for Medical Research, Heidelberg, Germany \\ ${ }^{2}$ Laboratorio de Plasticidad Cortical y Aprendizaje Perceptual, Instituto de Neurociencias, Universidad de Guadalajara, Guadalajara, México
}

\author{
Edited by: \\ Mathias V. Schmidt, Max Planck \\ Institute of Psychiatry, Germany \\ Reviewed by: \\ Allan V. Kalueff, International Stress \\ and Behavior Society and ZENERE \\ Institute, USA \\ Mathilakath Vijayan, University of \\ Waterloo, Canada \\ *Correspondence: \\ Rodrigo J. De Marco and Soojin \\ Ryu, Developmental Genetics of \\ the Nervous System, Max Planck \\ Institute for Medical Research, \\ Jahnstrasse 29, D-69120, \\ Heidelberg, Germany \\ e-mail: rodrigo.de.marco@ \\ mpimf-heidelberg.mpg.de; \\ soojin.ryu@ \\ mpimf-heidelberg.mpg.de
}

The relationship between stress and food consumption has been well documented in adults but less so in developing vertebrates. Here we demonstrate that an encounter with a stressor can suppress food consumption in larval zebrafish. Furthermore, we provide indication that food intake suppression cannot be accounted for by changes in locomotion, oxygen consumption and visual responses, as they remain unaffected after exposure to a potent stressor. We also show that feeding reoccurs when basal levels of cortisol (stress hormone in humans and teleosts) are re-established. The results present evidence that the onset of stress can switch off the drive for feeding very early in vertebrate development, and add a novel endpoint for analyses of metabolic and behavioral disorders in an organism suitable for high-throughput genetics and non-invasive brain imaging.

Keywords: stress response, HPA-axis, feeding, larval zebrafish, behavior

\section{INTRODUCTION}

Organisms respond to threats by activating a set of processes collectively referred to as the stress response (Selye, 1956; Chrousos, 1998). These processes aim to preserve body homeostasis and rely heavily on the hypothalamic-pituitary-adrenal (HPA) axis (Charmandari et al., 2005). The human hypothalamus contains groups of neurons concerned with the regulation of energy balance, sexual drive and sleep. It responds quickly to a state of threatened homeostasis, or stress, by triggering the release of key hormones from the pituitary, such as adrenocorticotropic hormone (ACTH; Sapolsky, 2000). The adrenal gland (interrenal gland in teleosts) then secretes cortisol under the control of $\mathrm{ACTH}$, released from the pituitary in response to signals from the hypothalamus, such as corticotropin-releasing-hormone $(\mathrm{CRH})$. Thus, external stimuli triggering the release of $\mathrm{CRH}$, $\mathrm{ACTH}$ and cortisol are termed stressors, and can exert various effects on behavior. Under non-stressful conditions, for example, food intake and energy expenditure are kept balanced through a process called energy homeostasis (Kennedy, 1953), controled by neurons in the hypothalamus and other brain areas receptive to peripheral metabolic signals (Gao and Horvath, 2007; Morton et al., 2014). Once the HPA-axis has been activated, however, a state of reduced appetite suppresses the normal regulation of energy homeostasis and prevents feeding until stress has ceased (Morton et al., 2014). This phenomenon involves actions by neurons in the hypothalamus and other brain areas (Morton et al., 2014), including the parabrachial nucleus (Carter et al., 2013), and a number of stress mediators, including glucocorticoids (Spina et al., 1996; Carr, 2002; Maniam and Morris, 2012;
Matsuda et al., 2013). So far, evidence linking acute stress to reduced appetite has been documented in adults (Carr, 2002), but less so in developing vertebrates. Here, we searched for direct evidence of stressor-mediated anorexia in larval zebrafish (Danio rerio).

Because of the ease of handling and their physiological and neuroanatomical homology to humans, zebrafish are becoming increasingly popular in biomedical research and models for behavioral and metabolic disorders are being established (Nguyen et al., 2013). In particular, larval zebrafish appear highly suitable for addressing mechanisms governing stress-induced anorexia. The zebrafish hypothalamic-pituitary-interrenal (HPI) axis shares conspicuous homologies with the HPA-axis in humans (Wendelaar Bonga, 1997; Löhr and Hammerschmidt, 2011), and the larvae's neurosecretory preoptic-hypothalamic area is comparable to the hypothalamic paraventricular nucleus in mammals (Herget et al., 2014). Also, measures of hormone levels and gene expression analysis show that the stress response can be triggered around 4 days post fertilization (dpf; Alsop and Vijayan, 2008, 2009a,b; Alderman and Bernier, 2009; Steenbergen et al., 2011; Yeh et al., 2013). Further, the energy reservoir (yolk sack) of embryos begins to deplete around $3 \mathrm{dpf}$ (Kimmel et al., 1995), and, by $6 \mathrm{dpf}$, larvae execute elaborate prey capture behaviors (Borla et al., 2002; McElligott and O'malley, 2005; Trivedi and Bollmann, 2013). Also importantly, due to their small size, genetic access and transparent body, larval zebrafish are suitable for high-throughput behavioral genetics, non-invasive brain imaging and optogenetic probing of neural circuits (Gahtan and Baier, 2004; Portugues et al., 2013). Altogether, these features favour the 
view that zebrafish larvae may contribute significantly to the study of how stress influences appetite and food consumption, although whether they are subject to stress-induced anorexia remains still unresolved.

Here we show that first encounters with distinct stressors can suppress feeding in larval zebrafish. Using computer-visionbased methods, we first developed a protocol for assessing food consumption in groups of freely swimming larvae. For this, we quantified the larvae's use of space in an environment with unevenly distributed prey, and validated our measure as an accurate assessment of food consumption. Next, we presented larvae with a well-established, albeit artificial stressor (i.e., hyperosmotic medium) and determined the relationship between ensuing whole-body cortisol and the larvae's feeding performance. We observed a robust stressor-mediated suppression of feeding. We then examined if such form of food intake suppression could be accounted for by altered locomotion, oxygen consumption or visual reactions. The results showed that it could not, favouring the view that the onset of stress acted on the larvae's drive for feeding. Finally, in order to ponder the link between stressor identity and stressor-mediated food intake suppression, we also tested feeding in larvae pre-exposed to a novel stress protocol based exclusively on water motions likely to occur in the zebrafish native environment, such as those originating from hovering fish or approaching predators. The results of these tests confirmed that initial stress and reduced feeding appear linked to each other. Also importantly, we found that feeding in stressed larvae reoccurred when basal cortisol levels were re-established. In sum, we found that larval zebrafish display a robust form of stressor-mediated anorexia and introduced a novel endpoint for analyses of stress effects on metabolic and behavioral disorders.

\section{MATERIAL AND METHODS ZEBRAFISH HUSBANDRY}

Zebrafish breeding and maintenance was performed under standard conditions (Westerfield, 2000). Embryos were collected in the morning and raised on a 12:12 light/dark cycle in E2 medium (Westerfield, 2000). All experiments were carried out with wildtype (cross between $\mathrm{AB}$ and TL strains) $6 \mathrm{dpf}$ larvae. Zebrafish experimental procedures were performed according to the guidelines of the German animal welfare law and approved by the local government.

\section{SALT STRESS}

Larvae were incubated for $10 \mathrm{~min}$ in steady state E2, E2 + $50 \mathrm{mM}$ $\mathrm{NaCl}\left(\mathrm{NaCl}_{50 \mathrm{mM}}\right)$ or $\mathrm{E} 2+100 \mathrm{mM} \mathrm{NaCl}\left(\mathrm{NaCl}_{100 \mathrm{mM}}\right)$ media, at $28^{\circ} \mathrm{C}$ under white light illumination. They were then washed three times with E2 medium and kept in a small container for oxygen consumption measurements (6-8 min later), or transferred to a custom made swimming chamber for behavioral testing. The wash and transfer period took $3 \mathrm{~min}( \pm 10 \mathrm{~s})$ and was performed at room temperature. Behavioral recordings began 5 min after stressor exposure. Cortisol detection was carried out using a home-made cortisol ELISA protocol, as described in (Yeh et al., 2013).

\section{MECHANOSENSORY STRESS}

Larvae were presented with fast hydrodynamic flows caused by the rapid lateral displacements (LDs) of a partially submerged inflexible silica capillary tube (Polymicro Technologies, AZ, $360 \mu \mathrm{m}$ OD, Optronis GmbH; Kehl, Germany) fixed to a multilayer piezo bender actuator (PICMA ${ }^{\circledR}$ PL140.10, Physik Instrumente (PI) GmbH and Co. KG; Karlsruhe, Germany). The actuator had an operating voltage of $0-60 \mathrm{~V}$, a maximum displacement of $\pm 1000 \mu \mathrm{m}$, and an unloaded resonant frequency of $160 \mathrm{~Hz}$. The bender was connected to a dual-piezo-amplifier (maximum voltage: $10 \mathrm{~V}$ ), a pulse generator and a TTL control system allowing for computer control. The tip of the silica capillary tube was placed, partially submerged $(2 \mathrm{~mm})$, at the center of a $35 \mathrm{~mm}$ petri dish half filled $(1.8 \mathrm{ml})$ with $\mathrm{E} 2$ medium (orientation relative to water surface: $90^{\circ}$ ). The voltage applied to the bender $\left(\mathrm{V}_{\mathrm{act}}\right)$ determined the speed of the capillary's LDs, or stimulus strength (in \% relative to maximum voltage). Groups of 30 larvae were exposed to 6 stimulation units delivered with an inter-stimulation-interval of $250 \mathrm{~ms}$. Each unit consisted of $9940 \mathrm{~ms}$ LDs. We used a $\mathrm{V}_{\text {act }}$ of 3, 4.5 or $6 \mathrm{~V}$. Stimulations were carried out under white illumination at $25^{\circ} \mathrm{C}$. After stimulation, larvae were kept in the petri dishes for cortisol measurement (9.5 min later) as described in (Yeh et al., 2013), or transferred to a custom made swimming chamber for behavioral testing, where they remained unperturbed for $10 \mathrm{~min}$ before recordings.

\section{OXYGEN CONSUMPTION}

Oxygen levels inside a single custom made flow chamber with 8 freely swimming larvae were monitored (as \% air saturated) every $5 \mathrm{~s}$ for $30 \mathrm{~min}$ using fibre optic sensing technology (PreSens Precision Sensing GmbH; Regensburg, Germany). To minimize perturbations during recordings, the chamber was placed inside a light-proof box. Its inlet and outlet channels had silicon tubes (750 $\mu \mathrm{m} \mathrm{OD)} \mathrm{and} \mathrm{a} 5 \mathrm{ml}$ Luer-Lok ${ }^{\mathrm{TM}}$ Tip Syringe (BD, Heidelberg, Germany) was connected to the inlet to fill the chamber with solution; both inlet and outlet remained closed during measurements. To calibrate the oxygen-sensitive sensor, we used oxygen-free (Cal 0) and air-saturated water (Cal 100). For Cal 0, $\mathrm{Na}_{2} \mathrm{SO}_{3}(0.2 \mathrm{~g})$ was dissolved in distilled water $(20 \mathrm{ml})$. For Cal 100, air was blown in distilled water $(100 \mathrm{ml})$ for $20 \mathrm{~min}$ while stirring; air flow was then stopped and the solution stirred for another $10 \mathrm{~min}$ to avoid hyper-saturation. We used a twopoint calibration with temperature and atmospheric pressure compensation. For each measurement, the oxygen consumption rate (OCR) was approximated as the slope of a linear fit to the oxygen level for $10 \mathrm{~min}<$ time $<30 \mathrm{~min}$. Prior to measuring the OCRs of larvae, the OCR of E2 alone was measured twice and averaged. Net OCRs of the larvae were calculated by subtracting the average OCR of E2 of the actual day from the OCRs of the larvae.

\section{BEHAVIORAL TESTING}

Experiments on feeding were conducted under both white and infrared (IR) light, delivered through a custom-made array of white- and IR-LEDs mounted inside a custom-made lightproof enclosure placed on a vibration-free platform (Newport, 
Irvine, CA, USA). Avoidance and individual locomotion tests were conducted under IR light only. In all cases, larvae were imaged at 25 frames $s^{-1}$ through an infrared-sensitive camera (ICD-49E B/W; Ikegami Tsushinki Co., Ltd. Japan) with its lens (TV Lens, Computer VARI FOCAL H3Z4512 CS-IR, CBC; Commak, NY, USA) positioned above a swimming chamber. We used EthoVision XT software (Noldus Information Technology; Wageningen, The Netherlands) and algorithms written in MATLAB 7.8 (MathWorks, Inc.; Natick, MA, USA) to monitor the movements of larvae swimming either individually (avoidance and individual locomotion tests) or in groups (feeding) inside custom-made chambers, respectively. In all experiments, larvae were allowed to adjust to the chamber conditions for several minutes prior to recording. Experiments were conducted at $28 \pm 1{ }^{\circ} \mathrm{C}$. A thermocouple (npi electronics $\mathrm{GmbH}$; Tamm, Germany) connected to a temperature control system (PTC 20, npi electronics GmbH; Tamm, Germany; Exos-2 V2 liquid cooling system, Koolance; Auburn, WA, USA) monitored the temperature inside the chambers. All the experiments were performed in a blind fashion as to group identity. Control animals for each group were handled in the same fashion, but omitting stimulus presentation. Tests were conducted between 9:00 and 18:00 and different experimental groups intermixed throughout the day.

\section{Feeding test}

Groups of twenty larvae pre-exposed to either E2 medium alone or salt or mechanosensory stress were placed in a custom-made swimming chamber (length: $40 \mathrm{~mm}$, width: $20 \mathrm{~mm}$, height: $10 \mathrm{~mm}$ ). Two vertically-oriented transparent walls (width: 500 $\mu \mathrm{m}$ ) divided the chamber into three contiguous compartments, S1, Centre and S2, with the Centre compartment being twice as large as each of the side compartments. The transparent walls divided only the upper part (eight tenth) of the cross section of the chamber, leaving space at the bottom $(2 \mathrm{~mm})$ for the larvae to move across compartments while a camera placed above recorded their movements under white light illumination. The walls also had contiguous rows of equidistant $100 \mu \mathrm{m}$ openings allowing the medium to diffuse. Also, they acted as barriers to confine paramecia to a single compartment, either S1 or S2. Their size, slow motion and tendency to remain in the upper part of the water column prevented paramecia from moving from one compartment to another during the $600 \mathrm{~s}$ of each recording session. Following acclimation, video-recordings were made first in the absence of food items (initial $10 \mathrm{~min}$ ) and, then, after having added a known quantity of paramecia to one of the chamber's side compartments, either S1 or S2 (final $10 \mathrm{~min}$ ). When assessing food consumption, paramecia were counted before and after the final 10 min video-recordings. The resulting differential space use (DSU) values were calculated and compared across groups.

\section{Quantifying motion and space use}

To measure motion and space use, we used an algorithm written in MATLAB 2009b (MathWorks, Inc.; Natick, MA, USA) which detects the movements of larvae swimming within one or several areas of interest using the pixel-by-pixel mean squared error (m.s.e) of gray-scale transformed and adjusted images from consecutive video-frames, given by:

$$
\text { m.s.e. }=\frac{1}{N} \sum_{\text {pixel }=1}^{N}\left(\text { image }_{\text {frame,pixel }}-\text { image }_{\text {frame-1,pixel }}\right)^{2}
$$

where $\mathrm{N}$ corresponds to the total number of pixels of each frame. We confirmed that m.s.e. remained constant in empty chambers, and that m.s.e. changes were exclusively due to the movements of swimming larvae. Also importantly, in our experiments simultaneous m.s.e. data from multiple areas (e.g., compartments) could be parsed and m.s.e. remained insensitive to moving paramecia due to the limited spatial resolution of the camera. Larval zebrafish are highly sensitive to photic stimuli (Burgess and Granato, 2007a), and react to sharp transitions from darkness to light with stereotypic changes in locomotion (MacPhail et al., 2009). Measurements from experiments where single or groups of dark adapted larvae were exposed to consecutive squared pulses of light of varying length and strength validated the m.s.e. index as a highly sensitive motion detector. The m.s.e. index is also a derivative of the number of larvae swimming within a given area of interest. Independent measurements from experiments varying the number of larvae (min: 1, max: 10) swimming within a small cylindrical chamber (10 $\mathrm{mm}$ ID) showed that, even over a time period as short as $10 \mathrm{~s}$, the relationship between m.s.e. and the number of larvae could be well approximated by a linear regression ( $p<0.0001$, R-squared: 0.98), indicating that the m.s.e. index is sensitive to both the amount of motion and the number of swimming larvae.

\section{Reaction to illumination change}

Larvae pre-exposed to either E2 medium alone or a hyperosmotic medium $\left(\mathrm{NaCl}_{50 \mathrm{mM}}\right.$ or $\left.\mathrm{NaCl}_{100 \mathrm{mM}}\right)$ were placed individually in a custom-made rectangular chamber (length: $40 \mathrm{~mm}$, width: $20 \mathrm{~mm}$, height: $10 \mathrm{~mm}$ ) kept in darkness and filled with $3 \mathrm{ml}$ of E2 medium. Following a $10 \mathrm{~min}$ acclimation period, single larvae were imaged at 25 frames $\mathrm{s}^{-1}$ for $120 \mathrm{~s}$. Each video-recording consisted of an initial period of $60 \mathrm{~s}$ under IR illumination, followed by a $10 \mathrm{~s}$ square pulse of white light $\left(0.1 \mathrm{~mW}^{*} \mathrm{~cm}^{-2}\right)$ and a final 50 s period under IR illumination. EthoVision XT software (Noldus Information Technology; Wageningen, The Netherlands) was used to monitor the movements of the single larvae; group comparisons were based on their average swim velocity (calculated over $10 \mathrm{~s}$ ) before, during and after light exposure.

\section{Optomotor response}

Larvae pre-incubated in either E2 medium or a hyperosmotic medium $\left(\mathrm{NaCl}_{50 \mathrm{mM}}\right.$ or $\left.\mathrm{NaCl}_{100 \mathrm{mM}}\right)$ were placed individually in a rectangular chamber (length: $40 \mathrm{~mm}$, width: $20 \mathrm{~mm}$, height: $10 \mathrm{~mm}$ ) filled with $3 \mathrm{ml}$ of E2 medium and mounted directly above a computer screen horizontally oriented. The screen displayed visual stimuli created via an algorithm written in MATLAB 2009b (MathWorks, Inc.; Natick, MA, USA). Visual stimuli consisted of gray dots (displayed against a white background offering a light power of $0.5 \mathrm{~mW}^{*} \mathrm{~cm}^{-2}$ ) of variable contrast (\%), diameter (in degrees), velocity (in degrees $\mathrm{s}^{-1}$ ) and number, as defined at a viewing distance of $2 \mathrm{~mm}$. We used EthoVision XT software 
(Noldus Information Technology; Wageningen, The Netherlands) to monitor the movements of larvae swimming individually at 28 $\pm 5^{\circ} \mathrm{C}$. In each recording session, single larvae were first presented with stationary dots and allowed to swim freely for $60 \mathrm{~s}$. Next, as soon as they reach any of the chamber's sides (either left or right), the dots began to move towards the opposite side of the chamber. Under these circumstances, larvae would align themselves with the moving dots and begin to swim consistently towards the opposite side of the chamber. To quantify their optomotor response, we measured the time interval in between when they started to move together with the dots and when they reached the opposite side of the chamber (latency, in seconds). The effects of dot contrast, diameter, velocity and number were tested by means of independent recordings.

\section{STATISTICAL ANALYSIS}

All data are shown as single measurement points, mean and standard error of the mean (S.E.M.) or box-and-whisker plots. We used paired Student's $t$-tests (two-tailed) for two-group comparisons as well as ANOVAs, followed by Bonferroni's post-hoc tests, or Kruskal-wallis tests (if the data did not fulfill the assumptions of the ANOVA), followed by Dunn's tests, for multiple group comparisons. We also used linear and non-linear regression analysis. Analyses were carried out using MS-Excel (Microsoft; Redmond, WA, USA), MATLAB 2009b (MathWorks, Inc.; Natick, MA, USA), Prism 5, (Graphpad Software Inc.; San Diego, CA, USA), Sigma Plot (Systat Software Inc.; San Jose, CA, USA), $\mathrm{R}$ (Freeware) and Virtual Dub (Freeware).

\section{RESULTS \\ MEASURING FOOD CONSUMPTION IN GROUPS OF FREELY SWIMMING LARVAE}

Twenty larvae were placed in a custom made swimming chamber where two transparent walls created three contiguous compartments: S1, Centre and S2 (Figure 1A). The Centre compartment, twice as large as the side compartments, acted as a transition zone between S1 and S2. The transparent walls divided only the upper part of the chamber's cross section, leaving space at the bottom for the larvae to move freely across compartments while a camera placed above recorded their movements under white light illumination. The walls contained rows of small openings allowing the medium to diffuse and acted as barriers to confine moving prey (paramecia), or food items, to a single side compartment, either S1 or S2 (see Methods). Each recording session lasted $600 \mathrm{~s}$. Larvae were allowed $10 \mathrm{~min}$ of acclimation before recordings started. Following acclimation, video-recordings were made first in the absence of food items (initial $600 \mathrm{~s}$ ) and, then, after having added a known quantity of paramecia to one of the chamber's side compartments, either S1 or S2 (final $600 \mathrm{~s}$ ). In the absence of prey and external stimuli (other than visual and hydrodynamic cues caused by their own movements), larvae spent equal amounts of time in S1 and S2 (Figure 1B, left: Paired $t$-test, $\left.t_{(10)}=0.1, p=0.92\right)$. On average, the percentage of larvae found in either S1 or S2 was $\sim 25 \%$, as expected from a symmetric distribution of individuals within the chamber (Figure 1B, right). This changed when paramecia were added to one of the side compartments, either S1 or S2 (Figure 1C).
The presence of prey reliably triggered a differential space use (DSU) by the larvae. They spent more time in the compartment with paramecia (Figure 1D, left: Paired $t$-test, $t_{(10)}=5.8$, $p=0.0002)$, increasing the number of individuals in it after $600 \mathrm{~s}$ (Figure 1D, right). To quantify the overall motion and the number of larvae swimming in specific areas of the chamber, we used a tracking algorithm which computes the pixelby-pixel m.s.e. between images from consecutive video-frames (Figures 1E,F, see also Methods). First, we calculated the area under the curve (a.u.c.) from ensued m.s.e. values (in $600 \mathrm{~s}$ ) in order to estimate the amount of larvae that swam concurrently within each of the chamber's compartments. Using these values, we then calculated a global measure of DSU, as the difference (in \%) between the a.u.c. values from $S 1$ and $S 2$, or $\left[\left(S 1_{\text {a.u.c. }}-\right.\right.$ $\mathrm{S} 2$ a.u.c. $\left.) /\left(\mathrm{S} 1_{\text {a.u.c. }}+\mathrm{S} 2_{\text {a.u.c. }}\right)\right]^{*} 100$, always using data from the side with prey as S1. A comparison of DSU values recorded with and without paramecia validated the approach as an accurate method for quantifying the relation between the spatial distributions of both prey and larvae (Figure 1G, Paired $t$-test, $t_{(9)}=10.8$, $p<0.0001)$. Importantly, by counting the number of paramecia consumed by the group of larvae (Figure $\mathbf{1} \mathbf{H}$, Paired $t$-test, $t_{(9)}=$ $17.2, p<0.0001$ ), we observed that DSU variability accounted for $97 \%$ of the variance of prey consumption (Figure 1H, Pearson's correlation, $p<0.0001)$. Further, a comparison of total a.u.c. values (i.e., summed values from S1, Centre and S2) showed that prey-dependent DSU changes could not be accounted for by changes in the overall level of motion displayed by larvae throughout the recording periods (Paired $t$-test, $t_{(9)}=0.21, p=0.84$ ).

\section{EXPOSURE TO A HYPEROSMOTIC MEDIUM TRIGGERS HPI-AXIS ACTIVATION}

Salt exposure is a well-established stressor in teleosts and has been shown to increase whole-body cortisol in larval zebrafish (Yeh et al., 2013). We observed that freely swimming larvae avoided a sudden increase of salt concentration, as they rapidly moved away from a spot where $5 \mu \mathrm{l}$ of $50 \mathrm{mM} \mathrm{NaCl}$ solution was added to their medium and continued to avoid the vicinity of the addition spot over a $180 \mathrm{~s}$ video-recording period; such a series of avoidance reactions did not occur when $5 \mu$ l of E2 were added to the medium (Figure 2A). In separate experiments, we next measured whole-body cortisol as a function of (1) $\mathrm{NaCl}$ concentration in a steady-state hyperosmotic medium, (2) $\mathrm{NaCl}$ exposure duration; and (3) time after exposure (Figures 2B-D). The results showed that $\mathrm{NaCl}$ exposure increased cortisol in a concentrationdependent manner (Figure 2B, Kruskal-Wallis test, $H=48.6$, $p<0.0001$, followed by Dunn's multiple comparison tests and linear regression), that maximum cortisol levels were detectable 5-10 min after $\mathrm{NaCl}$ exposure (Figure 2C, One-Way ANOVA, $F_{(4,29)}=22.8, p<0.0001$, followed by Bonferroni post-tests for pair comparisons and non-linear regression), and that basal levels were re-established $30 \mathrm{~min}$ later (Figure 2D, One-Way ANOVA, $F_{(3,23)}=25.4, p<0.0001$, followed by Bonferroni post-tests for pair comparisons and non-linear regression).

\section{SALT STRESS SUPPRESSES FEEDING}

To investigate how HPI-axis activation influences feeding in freely swimming larvae, we measured prey-dependent DSU changes 


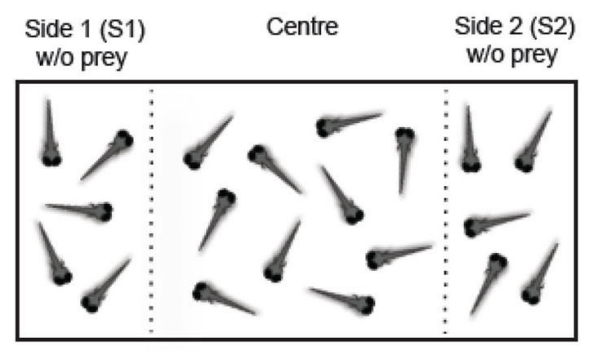

B

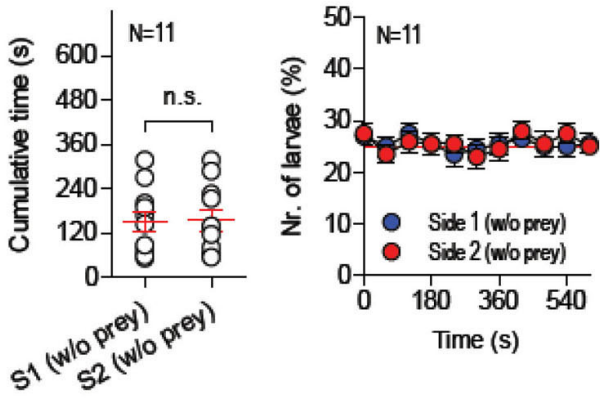

E
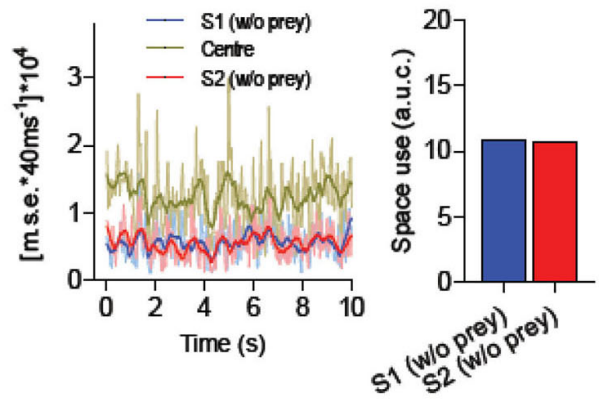

G

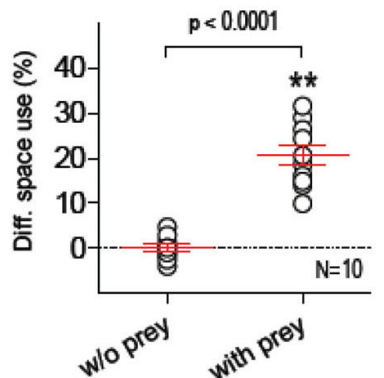

C
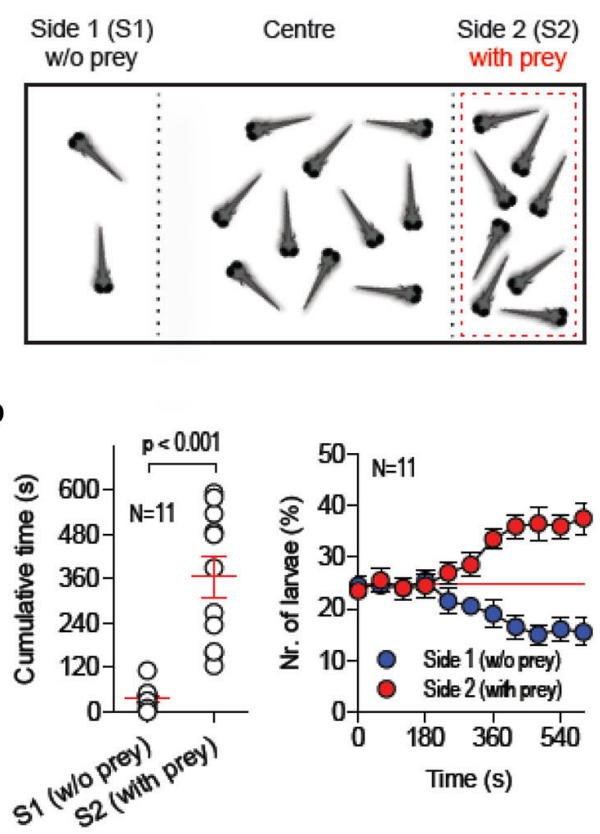

$\mathbf{F}$

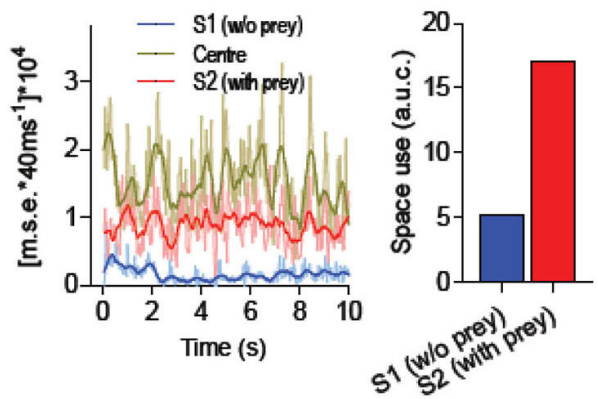

I

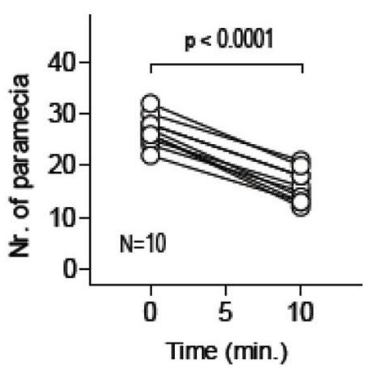

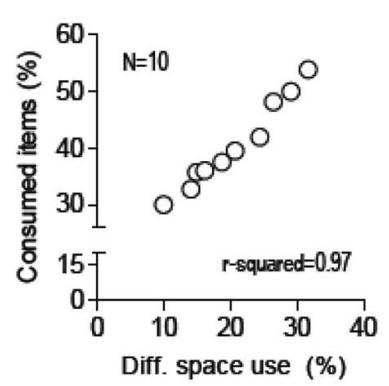

FIGURE 1 | Measuring food consumption in groups of freely swimming larvae. (A-C) Schematic of the basic experimental procedure: 20 freely behaving larvae are placed in a custom-made swimming chamber composed of three interconnected compartments (S1, Centre and S2). A camera placed above monitors their movements under white light illumination during two consecutive 10 min periods, first in the absence of food items (A, initial 10 $\mathrm{min}$ ) and then in the presence of a defined quantity of paramecia (prey) in one of the chamber's side compartments, either S1 or S2 (C), final $10 \mathrm{~min}$, prey in S2). The position of each larva and the overall activity of the group in S1. Centre and S2 are recorded. (B,D) Time spent by each larva and average percentage of larvae in S1 and S2 in the absence (B) and presence (D) of prey. $\mathbf{( E , F ) ~ E x e m p l a r y ~} 10 \mathrm{~s}$ traces of [mean squared error* $\left.40 \mathrm{~ms}^{-1}\right]^{*} 10^{4}(\mathrm{a}$ measure of global motion matching the number of swimming larvae) for each of the chamber's compartments (left), and resulting 'space use' (right, i.e., area under the curve from S1- and S2-traces) in the absence (E) and presence (F) of prey (see Methods). (G) Differential space use (DSU, in \%), determined as "space use" in S2 relative to that in S1, with and without prey (asterisks designate results from a one-sample $t$-test against $0, p<0.01$ ). (H) Number of paramecia in "side with prey" (either S1 or S2) at the beginning and the end of the final 10 min recording period. (I) DSU variance accounts for $97 \%$ of the variance of consumed paramecia (in \%, relative to the total amount of paramecia at the beginning of the 10 min session). 
A

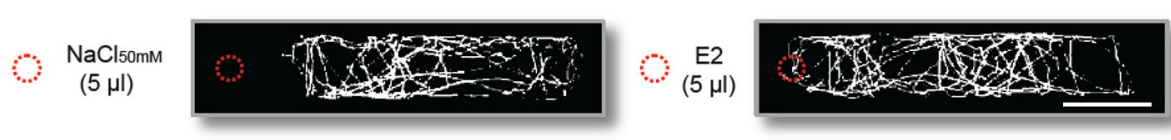

B

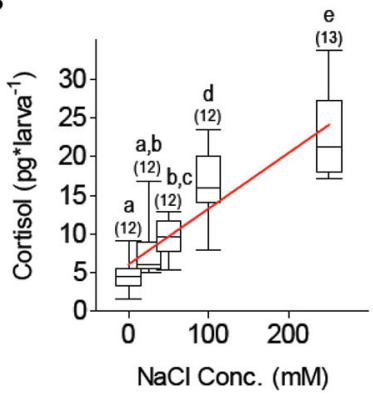

C

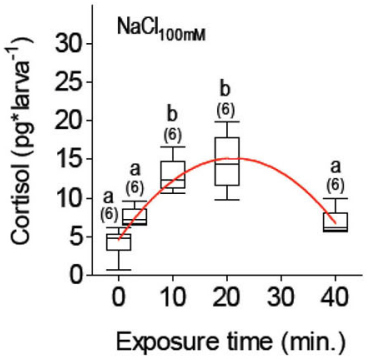

D

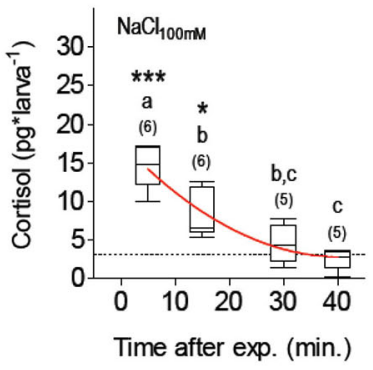

E

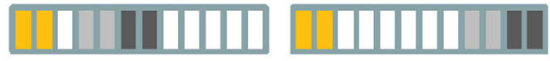

$\mathbf{F}$

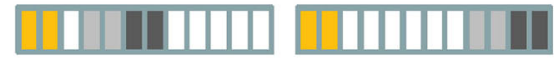

FIGURE 2 | Salt stress suppresses feeding. (A) Exemplary tracks from larvae swimming in darkness at constant temperature (scale bar: $10 \mathrm{~mm}$ ). Traces from single larvae appear biasedly distributed when $5 \mu$ l of $\mathrm{NaCl}_{50 \mathrm{mM}}$ (left), but not of E2 (right), are added (dashed red circle) to the $3 \mathrm{ml}$ E2 medium in the chamber, revealing active avoidance of sharp salinity variations. (B) $\mathrm{NaCl}$ exposure increases whole-body cortisol in a dose-dependent manner; linear regression $(p<0.0001)$ designated by red line. (C) Maximum cortisol levels are detected 10-20 min after $\mathrm{NaCl}$ exposure; as exposure length increases, cortisol drops after reaching a peak; non-linear regression (R-square $=0.78$ ) designated by red line. (D) Stress-induced cortisol reach

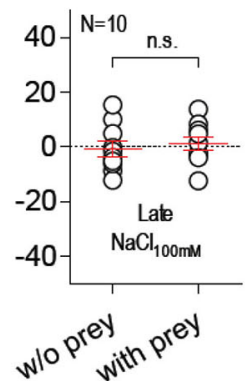

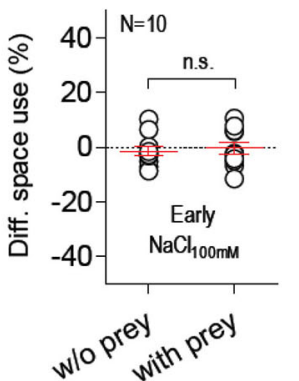

basal levels 30 min after $\mathrm{NaCl}$ exposure (dashed line denotes average basal levels); non-linear regression (R-square $=0.78$ ) designated by red line. (B-D: different letters designate statistical differences determined by one-way ANOVAs followed by post-hoc comparisons. Sample size in parentheses). (E,F) DSU in larvae pre-incubated with either $\mathrm{NaCl}_{50 \mathrm{mM}}$ (E) or $\mathrm{NaCl}_{100 \mathrm{mM}}$ (F). Top bars: each rectangle represents a 5 min time period; from left: $\mathrm{NaCl}$ exposure (yellow), first 10 min period without prey (light gray), second 10 min period with prey (dark gray). Shown are DSU values measured either 5-25 min (left, early) or 30-50 min (right, late) after $\mathrm{NaCl}$ exposure. Asterisks designate results from a one-sample $t$-test against $0, p<0.01$. in larvae that had been previously exposed (for $10 \mathrm{~min}$ ) to hyperosmotic $\mathrm{E} 2$ media containing additional $\mathrm{NaCl}\left(\mathrm{NaCl}_{50 \mathrm{mM}}\right.$ or $\left.\mathrm{NaCl}_{100 \mathrm{mM}}\right)$. Both incubations $\left(\mathrm{NaCl}_{50 \mathrm{mM}}\right.$ and $\left.\mathrm{NaCl}_{100 \mathrm{mM}}\right)$ suppressed prey-dependent DSU changes 15-25 min after the offset of $\mathrm{NaCl}$ exposure (Figures 2E,F, left, Paired $t$-tests, $\mathrm{NaCl}_{50 \mathrm{mM}}$ : $\left.t_{(9)}=0.51, p=0.62, \mathrm{NaCl}_{100 \mathrm{mM}}: t_{(9)}=0.38, p=0.72\right)$. Later, 40-50 min after exposure, prey-dependent DSU changes were detectable in larvae pre-incubated with $\mathrm{NaCl}_{50 \mathrm{mM}}$ but not with $\mathrm{NaCl}_{100 \mathrm{mM}}$ (Figures 2E,F, right, Paired $t$-tests, $\mathrm{NaCl}_{50 \mathrm{mM}}$ : $\left.t_{(9)}=4.02, p=0.003, \mathrm{NaCl}_{100 \mathrm{mM}}: t_{(9)}=0.51, p=0.63\right)$. Larvae pre-incubated with $\mathrm{NaCl}_{100 \mathrm{mM}}$ showed normal prey-dependent DSU changes, i.e., indistinguishable from those of control larvae, three hours after $\mathrm{NaCl}$ exposure (Paired $t$-test, $t_{(9)}=4.3$, $p=0.002$ ). Control larvae (pre-incubated with normal E2 medium) showed prey-dependent DSU changes 15-25, 40-50 and 180-190 min after incubation (Paired $t$-tests, 15-25 min: $t_{(9)}=8.72, p<0.0001,40-50 \mathrm{~min}: t_{(9)}=7.1, p<0.0001$, 180-190 min: $\left.t_{(9)}=4.6, p=0.001\right)$. A comparison of the total a.u.c. values from all groups indicated that motion variability could not account for the observed prey-dependent changes in DSU (Two-Way Repeated-Measures ANOVA, Group factor: $F_{(7,72)}=0.56, p=0.78$, Prey factor: $F_{(1,72)}=0.21, p=0.65$, Group X Prey factor: $\left.F_{(7,72)}=0.12, p=0.99\right)$.

\section{LOCOMOTION, OXYGEN CONSUMPTION AND VISUAL RESPONSES REMAIN UNALTERED AFTER STRESSOR EXPOSURE}

We next asked whether altered locomotion, oxygen consumption or visual reactions could account for the differences in preydependent space use observed after $\mathrm{NaCl}$ exposure. We first monitored post-exposure levels of locomotor activity in treated and 
control larvae within a stimulus-deprived environment. For this, larvae pre-incubated in either control or hyperosmotic mediums were placed in a custom-made, vibration-free swimming chamber (10 mm ID, volume: $400 \mu \mathrm{l}$ ) and allowed to swim individually in complete darkness for $600 \mathrm{~s}$ under constant temperature (28 $\pm 0.1^{\circ} \mathrm{C}$ ). We found that $\mathrm{NaCl}$ exposure failed to modify postexposure locomotion (Figure 3A, One-Way ANOVA, $F_{(2,71)}=$ 2.2, $p=0.13)$; as a result, peak cortisol levels after salt exposure did not account for locomotion variability (Figure 3B, Pearson's correlation, $p=0.42$ ). To expand the analysis of $\mathrm{NaCl}$ effects, we monitored oxygen levels every $5 \mathrm{~s}$ for $30 \mathrm{~min}$ (as \% of air saturated) in a custom-made chamber containing groups of eight freely swimming larvae that had, or had not, been preincubated in a hyperosmotic medium (of either $\mathrm{NaCl}_{50 \mathrm{mM}}$ or $\mathrm{NaCl}_{100 \mathrm{mM}}$ ). For each measurement, the OCR was approximated as the slope of a linear fit to the oxygen level for $10 \mathrm{~min}<$ time $<30 \mathrm{~min}$. Prior to measuring the OCRs of larvae, we measured (twice) the OCR of E2 medium alone. Net OCRs were calculated by subtracting the average OCR of E2 from the OCRs of the larvae. We found that pre-incubation with either $\mathrm{NaCl}_{50 \mathrm{mM}}$ or $\mathrm{NaCl}_{100 \mathrm{mM}}$ did not change net OCRs, as compared to pre-incubation with E2 (Figure 3C, One-Way ANOVA, $\left.F_{(2,23)}=0.26, p=0.77\right)$. Next, because larvae rely on vision for detecting and capturing prey, we set up to detect possible effects of $\mathrm{NaCl}$ exposure on their reactions to illumination change and moving visual stimuli. First, we examined motion reactions of control and treated larvae to a sudden illumination change and observed that dark-adapted larvae of either group reacted similarly to a $10 \mathrm{~s}$ square pulse of white light $\left(0.1 \mathrm{~mW}^{*} \mathrm{~cm}^{-2}\right)$. They reduced their locomotor activity similarly when exposed to light (Figure 3D, Two-Way Repeated-Measures ANOVA, Group factor: $F_{(1,36)}=0.41, p=0.53$, Light factor: $F_{(2,36)}=391.7$, $p<0.0001$, Group X Light factor: $\left.F_{(2,36)}=0.69, p=0.51\right)$. Next, we tested the larvae's optomotor response, i.e., spontaneous swimming in the direction of large-field displacements in the visual field. For this we presented control and treated larvae with ventrally moving dots of variable contrast, diameter, velocity and number (Figure 3E, see Methods). The results showed that $\mathrm{NaCl}$ exposure did not impair the larvae's optomotor response. Figure 3F shows how dot contrast improved the larvae's response, and how the response of larvae pre-incubated in either $\mathrm{NaCl}_{50 \mathrm{mM}}$ or $\mathrm{NaCl}_{100 \mathrm{mM}}$ did not differ from that of control larvae (TwoWay ANOVA, Group factor: $F_{(2,54)}=2.49, p=0.09$, Contrast factor: $F_{(1,54)}=69.57, p<0.0001$, Group $\mathrm{X}$ Contrast factor: $\left.F_{(2,54)}=0.59, p=0.56\right)$. Similar results arose from varying the diameter, velocity and number of moving dots (data not shown). In sum, locomotion, oxygen consumption and responses to visual inputs appeared unaltered under conditions of elevated HPI-axis activity caused by exposure to hyperosmotic mediums.

\section{MECHANOSENSORY STRESS SUPPRESSES FEEDING}

We next examined the extent to which stressor identity accounted for the effect of $\mathrm{NaCl}$ exposure on food intake. We therefore measured prey-dependent DSU changes in larvae pre-exposed to a novel stress protocol based exclusively on mechanosensory stimuli. To evoke mechanosensory stress, we used fast water movements caused by the rapid LDs of an inflexible silica capillary (360 $\mu \mathrm{m}$ OD) submerged partially $(2 \mathrm{~mm})$ in the larvae's surrounding medium. The capillary was fixed to a computer-controled piezo bender actuator and the voltage applied to the actuator (or stimulus strength, in \% relative to maximum voltage) determined the speed of the capillary's LDs (maximum displacement: $\pm 1000 \mu \mathrm{m}$ ). To examine how larvae reacted to the capillary's LDs, we placed them individually in a rectangular swimming chamber and LDs of known frequency and duration were triggered only when they swam within the half of the chamber containing the tip of the capillary (stimulus source). Larvae responded to LDs by increasing rapidly their distance to the centre point of the moving capillary (Figure 4A). In line with this, their overall locomotion during continuous stimulation (i.e., series of 10 2-ms LDs delivered at $1 \mathrm{~Hz}$, irrespective of the larva's position relative to the source) increased together with stimulus strength (Figure 4B). We thus assumed that LD-borne stimuli resembled, at least partially, motion and pressure waves (cues) encoding predation threat, such as those derived from the movements of larger fish and approaching predators. Notably, such a form of repeated mechanosensory stimulation increased whole-body cortisol in a stimulus strength-dependent manner (Figure 4C, Kruskal-Wallis test, $H=22.4, p<0.0001$, followed by Dunn's multiple comparison tests and linear regression), and post-peak cortisol levels were undistinguishable from basal levels $30 \mathrm{~min}$ after stimulation (Figure 4D, Kruskal-Wallis test, $H=10.8$, $p=0.0045$, followed by Dunn's multiple comparison tests and non-linear regression). An analysis of space use after mechanosensory stimulation showed that low (30\%) and moderate $(60 \%)$ stimulus strength levels abolished preydependent DSU changes 15-25 min after exposure to LDs (Figures 4E,F, left, Paired $t$-tests, 30\%: $t_{(23)}=0.48, p=0.63,60 \%$ : $\left.t_{(23)}=0.004, p=0.99\right)$. Normal DSU changes were detectable 40 $50 \mathrm{~min}$ later (Figures 4E,F, right, Paired $t$-tests, 30\%: $t_{(23)}=4.2$, $\left.p=0.0004,60 \%: t_{(23)}=4.6, p=0.0001\right)$. As before, across group comparisons of total a.u.c. values indicated that locomotion variability could not account for prey-dependent DSU changes (TwoWay Repeated-Measures ANOVA, Group factor: $F_{(5,54)}=0.38$, $p=0.86$, Prey factor: $F_{(1,54)}=0.40, p=0.53$, Group X Prey factor: $\left.F_{(5,54)}=0.20, p=0.96\right)$.

\section{DISCUSSION}

The small size, genetic access and transparency of larval zebrafish make them suitable for probing brain elements and peripheral effectors governing processes counteracting stress. For example, optogenetically induced hypercortisolaemia (De Marco et al., 2013) can be used to specify the effect of enhanced pituitaryinterrenal responses on stressor-mediated anorexia. However, despite advancements in techniques for altering and measuring specific cell activity (Friedrich et al., 2010), meaningful endpoints reflecting the consequences of stressful events in zebrafish larvae have lagged behind. We show that larval zebrafish execute avoidance reactions to salinity variance and threatening water motions, that exposure to either hyperosmotic mediums or potent hydrodynamic stimuli increases whole-body cortisol in a stimulus strength-dependent manner, and that both such 
A

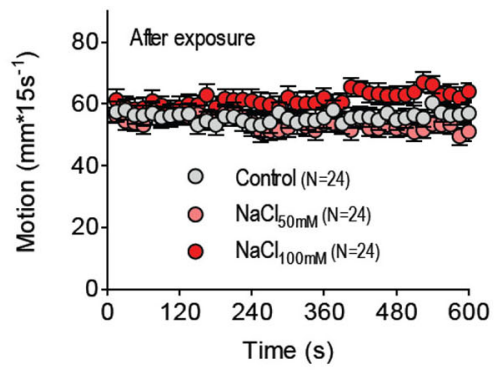

D

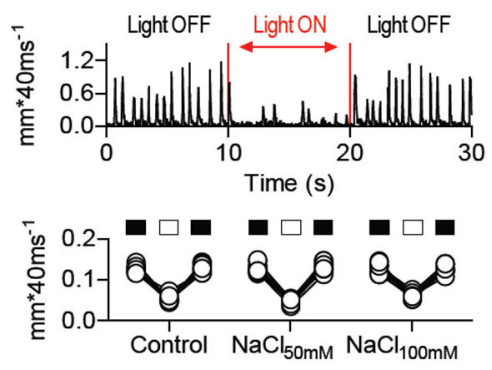

B

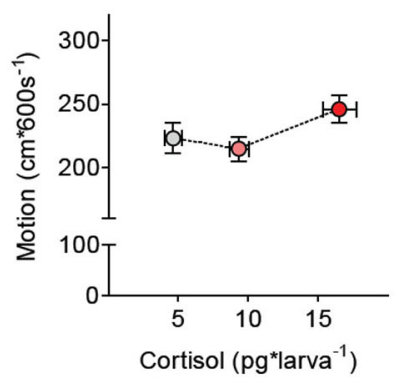

E

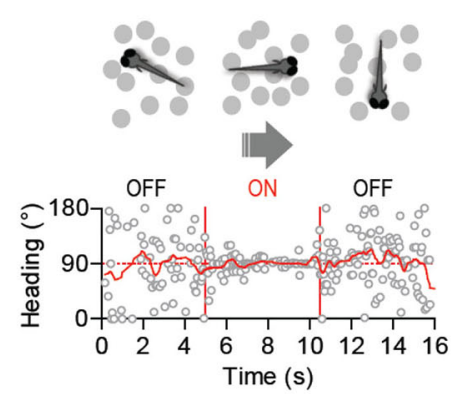

C

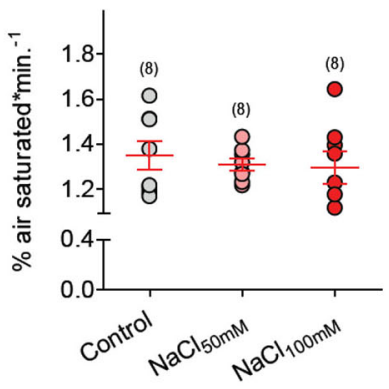

$\mathbf{F}$

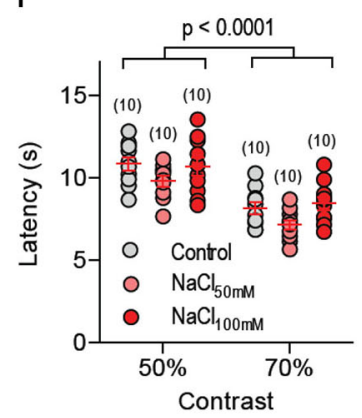

FIGURE 3 | Locomotion, oxygen consumption and visual responses remain unaltered after stressor stress. (A) Locomotor activity of larvae pre-incubated with $\mathrm{E2}, \mathrm{NaCl}_{50 \mathrm{mM}}$ and $\mathrm{NaCl}_{100 \mathrm{mM}}$ swimming individually in darkness at $28^{\circ} \mathrm{C}\left( \pm 0.1^{\circ} \mathrm{C}\right)$. (B) Average locomotion (over $600 \mathrm{~s}$ ) plotted against whole body cortisol for larvae pre-treated with $\mathrm{E} 2, \mathrm{NaCl}_{50 \mathrm{~mm}}$ and $\mathrm{NaCl}_{100 \mathrm{mM}}$. (C) Net oxygen consumption rates (OCRs) of groups of (eight) larvae pre-treated with $\mathrm{E} 2, \mathrm{NaCl}_{50 \mathrm{~mm}}$ or $\mathrm{NaCl}_{100 \mathrm{~mm}}$. (A,C: sample size in parentheses). (D) Swim velocity of single dark-adapted larvae pre-treated with $\mathrm{E} 2, \mathrm{NaCl}_{50 \mathrm{~mm}}$ or $\mathrm{NaCl}_{100 \mathrm{~mm}}$ before, during and after a $10 \mathrm{~s}$ square pulse of white light $\left(0.1 \mathrm{~mW}^{*} \mathrm{~cm}^{-2}\right)$. (E) Exemplary trace from an optomotor test depicting a larva's heading (relative to the long axis of a rectangular swimming chamber) as a function of time; during the test, single larvae are presented with gray dots (of variable contrast, diameter, velocity and number) ventrally displayed against a white background via a computer screen beneath the chamber (see Methods). No preferred heading can be detected when the dots remain stationary (OFF); by contrast, larvae swim in the direction of the moving dots when they move along the long axis of the chamber $(\mathrm{ON})$, thereby showing a preferred heading. Following acclimation and baseline recording, dot movements begin automatically when the larva is at one of chamber's sides (either left or right); dots move towards the chamber's opposite side and the time elapsed until the larva reaches the end of the chamber (latency) is taken as indicative of response strength. (F) Latency (s) from an optomotor test as a function of dot contrast for larvae pre-treated with $\mathrm{E} 2, \mathrm{NaCl}_{50 \mathrm{mM}}$ or $\mathrm{NaCl}_{100 \mathrm{mM}}$. treatments can lead to a full suppression of food consumption. Furthermore, we show that feeding reoccurs when basal cortisol levels are re-established.

If reversible, changes in responsiveness to external stimuli, such as those derived from living prey, are called motivational changes (McFarland, 1971), whereas sets of interacting elements controling groups of related activities (Tinbergen, 1951), such as those serving food search and feeding, are called motivational systems, with system variables defining states of increased or decreased hunger, for example. Understanding the basis of motivational change is a fundamental problem in stress research, where distinct behavioral categories are being influenced by assortments of hormones exerting pleiotropic actions over variable time periods (Morton et al., 2014). Nonetheless, the study of motivational change is challenged by the fact that systemic parameters and state variables can be influenced by a number of physiological processes, triggered, for example, by environmental stimuli, maturation and learning, a fact that introduces non-linear and non-stationary phenomena into the analysis (McFarland, 1971). For example, increased CRH levels can alter locomotion (Lowry and Moore, 2006) and, consequently, also food search activities. Likewise, stress mediators can interact with visuomotor pathways and modify the regulation of preycapture movements (Carr, 2002). We observed that an encounter with a potent stressor suppressed feeding without causing altered locomotion, oxygen consumption and visual reactions, indicating that the onset of stress can directly alter the larvae's motivation to feed, thus prompting a state stressor-mediated anorexia. Our observations are in line with the fact that, except upon initial contact with a stressor or under severe conditions of prolonged stress (Selye, 1956; Clark et al., 2011), tightly regulated bodily functions like motor control, energy balance and mechanisms for weighting external stimuli tend not to exceed their normal limits of variation (Selye, 1950; Teichner, 1968). The results are relevant for several reasons.

First, exposure to stressors and stress mediators have often been linked to food intake suppression in adults, but less so in developing vertebrates. The link between stress and reduced food consumption is well documented in adult teleosts, for example. Crowding and handling (Upton and Riley, 2013), as well as 
A

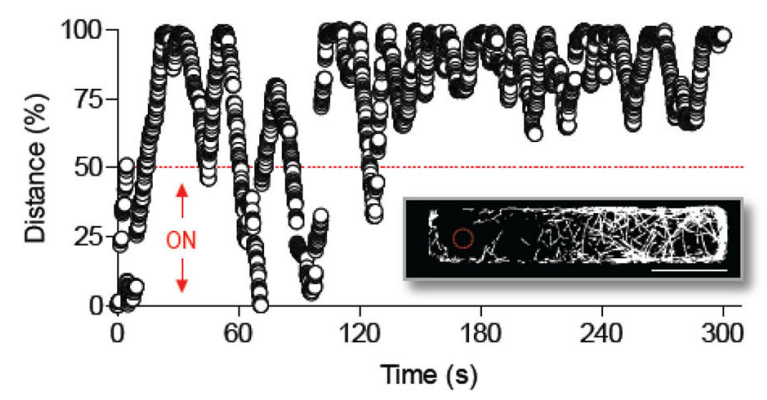

B

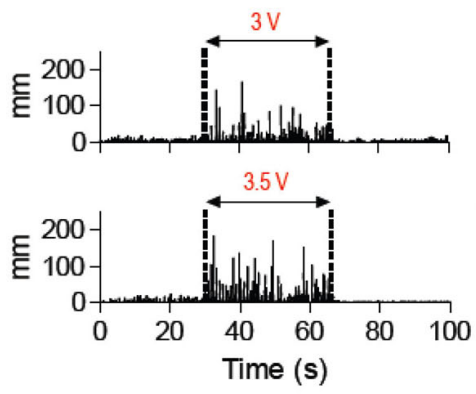

E

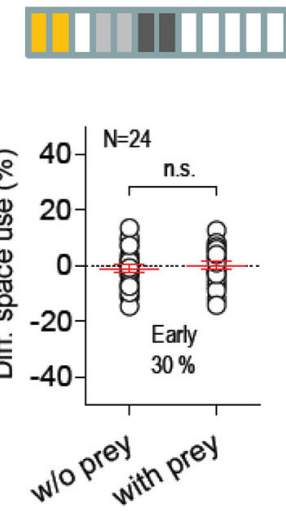

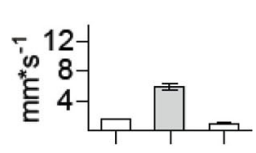

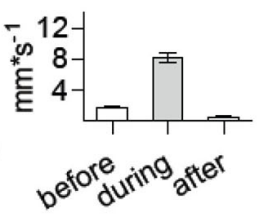

C

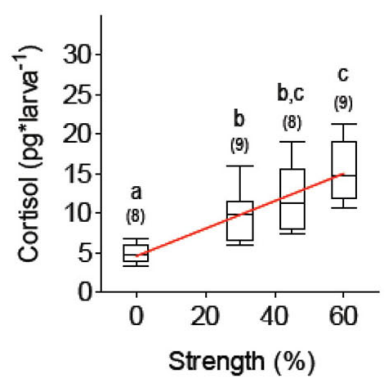

D

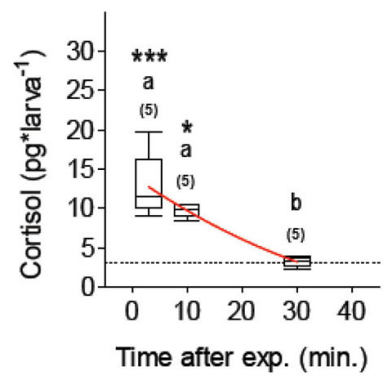

$\mathbf{F}$
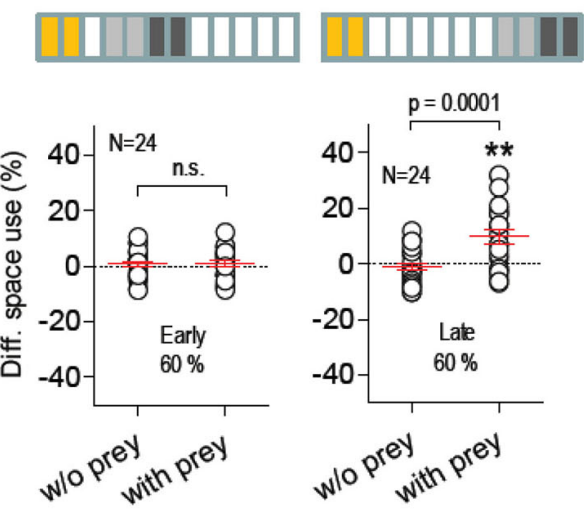

FIGURE 4 | Mechanosensory stress suppresses feeding. (A) Distance (in \% relative to maximum) every $40 \mathrm{~ms}$ between a single larva swimming in darkness at $28^{\circ} \mathrm{C}\left( \pm 0.1^{\circ} \mathrm{C}\right)$ and the submerged tip of a silica capillary tube fixed to a computer-controled piezo bender actuator. The capillary's tip (stimulus source) moves laterally upon voltage applied to the actuator, thereby causing fast hydrodynamic flows. The bender's LDs (of known frequency and duration) can be triggered at any given time or only when the larva swims within pre-defined areas of the swimming chamber, such as the half of the chamber containing the stimulus source (ON, bottom). Larvae respond to the stimulus by increasing the distance to the source. Insert: $x-y$ coordinates from an exemplary $300 \mathrm{~s}$ track illustrating how a single larva avoids the area surrounding the source (red dashed circle, scale bar, $10 \mathrm{~mm}$ ). (B) Left: distance swam by representative larvae before, during (between dashed lines) and after stimulation of increasing stimulus strength (delivered at $1 \mathrm{~Hz})$, as determined by the voltage applied to the bender $\left(\mathrm{V}_{\text {act }}\right)$, either 3 (top) or $3.5 \mathrm{~V}$ (bottom); right: average distance swam before, during and after stimulation. (C) Whole-body cortisol measured 10 min after stimulation as a function of stimulus strength (in \% relative to maximum $V_{\text {act }}$ ); linear regression ( $p<0.0001$ ) designated by red line. (D) Cortisol level as a function of time after stimulation (stimulus strength: 60\%). Asterisks designate statistical differences as compared to basal levels ( ${ }^{*} p<0.05,{ }^{* * *} p<0.001$ ). Non-linear regression ( $R$-square $=0.77$ ) designated by red line $(\mathbf{C}, \mathbf{D}$ : different letters designate statistical differences determined by one-way ANOVAs followed by post-hoc comparisons; sample size in parentheses). (E,F) DSU in larvae pre-exposed to mechanosensory stress using stimulus strength levels of $30 \%$ (E) and $60 \%(\mathbf{F})$, defined as in (C) (asterisks designate results from one-sample $t$-tests against $\left.0,{ }^{* *} p<0.01,{ }^{* * *} p<0.001\right)$. Top bars: each rectangle represents a 5 min time period; from left: mechanosensory stimulation (yellow), first 10 min period without prey (light gray), second 10 min period with prey (dark gray). Shown are DSU values measured either 5-25 min (left, early) or 30-50 min (right, late) after mechanosensory stimulation. cortisol administration (Janzen et al., 2012), have been shown to inhibit food intake in tilapia. Confinement caused elevated cortisol and suppressed feeding in salmon (Pankhurst et al.,
2008). Hypoxia (Bernier et al., 2012) and salt exposure (De Boeck et al., 2000) reduced food intake in common carp, and crowding (Conde-Sieira et al., 2010) and cortisol implants (Gregory and 
Wood, 1999) inhibited food consumption in rainbow trout. Also, social subordination has been found to abolish feeding in arctic char (Øverli et al., 1998), whereas CRH-injection impaired food intake in goldfish (de Pedro et al., 1997). Furthermore, CRF, urotensin I, and serotonin (5-HT) have recently been identified as anorexigenic agents in rainbow trout (Ortega et al., 2013). In adult zebrafish, pre-exposure to either aversive stimuli or alarm cues caused reduced feeding (Oswald and Robison, 2008). Also, just as in humans, where the effect of stress on food intake can be either positive or negative, models of both stress-induced anorexia and stress-induced obesity are being advanced (Merali et al., 2013), highlighting the complexity of the link between stress and metabolic disorders. It has been shown, for example, that adult zebrafish exposed to alarm signals gained weight (as compared to non-exposed individuals) only if they were on a high-fat diet (Nguyen et al., 2013). In developing vertebrates, including teleosts, only a few studies have related adverse conditions and stress hormones to reduced feeding. It has been shown, that $\mathrm{CRH}$ administration inhibited feeding in neonatal boiler chicks (Furuse et al., 1997), and that exogenous corticosterone increased the latency to beg for food and suppressed growth in white-crowed sparrow nestlings (Wada and Breuner, 2008). In both pre- and pro-metamorphic tadpoles, intracerebroventricular CRF injection inhibited food intake, and the CRF antagonist alpha-helical CRF suppressed food intake in pro-metamorphic tadpoles (Crespi and Denver, 2004, 2005). Also in tadpoles, altered rearing conditions (i.e., varying population density and food availability) increased corticosteroid levels, which appeared correlated to reduced growth (Glennemeier and Denver, 2002a,b). What has persistently been absent in developing vertebrates is evidence linking stressors to "appetite" (central drive) suppression. The results presented here provide first evidence for stressor-mediated anorexia occurring very early in vertebrate development.

Second, escape reactions to different forms of motion and pressure waves have long been reported in larval zebrafish (Kimmel et al., 1974; Zeddies and Fay, 2005; Burgess and Granato, 2007b; Best et al., 2008; McHenry et al., 2009; Roberts et al., 2011; Buck et al., 2012; Kohashi et al., 2012; Olszewski et al., 2012; Bhandiwad et al., 2013; Stewart et al., 2013). Our experiments introduce a novel stress protocol based on fast water motions resembling motion and pressure waves likely to occur in the zebrafish native environment, such as those caused by larger fish and approaching predators (Engeszer et al., 2007; Spence et al., 2008). Because varying levels of salt, $\mathrm{pH}, \mathrm{EtOH}$, heavy metals and severe swirling or vortexing have been shown to increase cortisol in larval zebrafish (Alsop and Vijayan, 2008, 2009a,b; Alderman and Bernier, 2009; Steenbergen et al., 2011; Yeh et al., 2013), the protocol described here will aid effort to compare effects of purely physical and more complex physico-chemical stressors.

Third, the results add a novel assay to the repertoire of laboratory tests of larval zebrafish, an organism increasingly popular in translational research that still lacks sufficient behavioral assessments. Larval zebrafish allow for in vivo analyses of brain function and behavior and may offer an excellent opportunity for dissecting short- and long-term effects of stressors on nervous and hormonal control systems. Adding to previous works, our data provide a behavioral endpoint suitable to be combined with pharmacological and genetic targeting of specific cells within the HPI-axis, as well as optogenetic circuit analysis, in order to examine metabolic and behavioral disorders.

\section{AUTHOR CONTRIBUTIONS}

Conception and design of the experiments: Rodrigo J. De Marco and Soojin Ryu. Acquisition of data: Rodrigo J. De Marco, Antonia H. Groneberg, Chen-Min Yeh. Development of MATLAB scripts: Mario Treviño. Analysis and interpretation of data: Rodrigo J. De Marco, Antonia H. Groneberg, Soojin Ryu. Writing the article: Rodrigo J. De Marco and Soojin Ryu.

\section{ACKNOWLEDGMENTS}

We thank Siegfried Hänselmann for assistance with experiments and K. Schmidt, R. Rödel, M. Lukat and N. Neef for logistic support. We thank Robert Denver for critical reading of the manuscript. Mario Treviño was supported by CONACyTMéxico ("Programa de Repatriación"). This work was supported by DFG-FOR1279, the Max Planck Society and Behrens-Weise Foundation.

\section{REFERENCES}

Alderman, S. L., and Bernier, N. J. (2009). Ontogeny of the corticotropin-releasing factor system in zebrafish. Gen. Comp. Endocrinol. 164, 61-69. doi: 10.1016/j. ygcen.2009.04.007

Alsop, D., and Vijayan, M. M. (2008). Development of the corticosteroid stress axis and receptor expression in zebrafish. Am. J. Physiol. Regul. Integr. Comp. Physiol. 294, R711-R719. doi: 10.1152/ajpregu.00671.2007

Alsop, D., and Vijayan, M. (2009a). The zebrafish stress axis: molecular fallout from the teleost-specific genome duplication event. Gen. Comp. Endocrinol. 161, 6266. doi: 10.1016/j.ygcen.2008.09.011

Alsop, D., and Vijayan, M. M. (2009b). Molecular programming of the corticosteroid stress axis during zebrafish development. Comp. Biochem. Physiol. A Mol. Integr. Physiol. 153, 49-54. doi: 10.1016/j.cbpa.2008.12.008

Bernier, N. J., Gorissen, M., and Flik, G. (2012). Differential effects of chronic hypoxia and feed restriction on the expression of leptin and its receptor, food intake regulation and the endocrine stress response in common carp. J. Exp. Biol. 215, 2273-2282. doi: 10.1242/jeb.066183

Best, J. D., Berghmans, S., Hunt, J. J., Clarke, S. C., Fleming, A., Goldsmith, P., et al. (2008). Non-associative learning in larval zebrafish. Neuropsychopharmacology 33, 1206-1215. doi: 10.1038/sj.npp.1301489

Bhandiwad, A. A., Zeddies, D. G., Raible, D. W., Rubel, E. W., and Sisneros, J. A. (2013). Auditory sensitivity of larval zebrafish (Danio rerio) measured using a behavioral prepulse inhibition assay. J. Exp. Biol. 216, 3504-3513. doi: 10. 1242/jeb.087635

Borla, M. A., Palecek, B., Budick, S., and O'malley, D. M. (2002). Prey capture by Larval Zebrafish: evidence for fine axial motor control. Brain Behav. Evol. 60, 207-229. doi: 10.1159/000066699

Buck, L. M., Winter, M. J., Redfern, W. S., and Whitfield, T. T. (2012). Ototoxininduced cellular damage in neuromasts disrupts lateral line function in larval zebrafish. Hear. Res. 284, 67-81. doi: 10.1016/j.heares.2011.12.001

Burgess, H. A., and Granato, M. (2007a). Modulation of locomotor activity in larval zebrafish during light adaptation. J. Exp. Biol. 210, 2526-2539. doi: 10.1242/jeb. 003939

Burgess, H. A., and Granato, M. (2007b). Sensorimotor gating in larval zebrafish. J. Neurosci. 27, 4984-4994. doi: 10.1523/jneurosci.0615-07.2007

Carr, J. A. (2002). Stress, neuropeptides and feeding behavior: a comparative perspective. Integr. Comp. Biol. 42, 582-590. doi: 10.1093/icb/42.3.582

Carter, M. E., Soden, M. E., Zweifel, L. S., and Palmiter, R. D. (2013). Genetic identification of a neural circuit that suppresses appetite. Nature 503, 111-114. doi: $10.1038 /$ nature 12596 
Charmandari, E., Tsigos, C., and Chrousos, G. (2005). Endocrinology of the stress response. Annu. Rev. Physiol. 67, 259-284. doi: 10.1146/annurev.physiol.67. 040403.120816

Chrousos, G. P. (1998). Stressors, stress and neuroendocrine integration of the adaptive response. The 1997 Hans Selye memorial lecture. Ann. N Y Acad. Sci. 851, 311-335. doi: 10.1111/j.1749-6632.1998.tb09006.x

Clark, K. J., Boczek, N. J., and Ekker, S. C. (2011). Stressing zebrafish for behavioral genetics. Rev. Neurosci. 22, 49-62. doi: 10.1515/rns.2011.007

Conde-Sieira, M., Aguilar, A. J., López-Patiño, M. A., Miguez, J. M., and Soengas, J. L. (2010). Stress alters food intake and glucosensing response in hypothalamus, hindbrain, liver and Brockmann bodies of rainbow trout. Physiol. Behav. 101, 483-493. doi: 10.1016/j.physbeh.2010.07.016

Crespi, E. J., and Denver, R. J. (2004). Ontogeny of corticotropin-releasing factor effects on locomotion and foraging in the Western spadefoot toad (Spea hammondii). Horm. Behav. 46, 399-410. doi: 10.1016/j.yhbeh.2004. 03.011

Crespi, E. J., and Denver, R. J. (2005). Roles of stress hormones in food intake regulation in anuran amphibians throughout the life cycle. Comp. Biochem. Physiol. A Mol. Integr. Physiol. 141, 381-390. doi: 10.1016/j.cbpb.2004.12.007

De Boeck, G., Vlaeminck, A., Van Der Linden, A., and Blust, R. (2000). The energy metabolism of common carp (Cyprinus carpio) when exposed to salt stress: an increase in energy expenditure or effects of starvation? Physiol. Biochem. Zool. 73, 102-111. doi: 10.1086/316717

De Marco, R. J., Groneberg, A. H., Yeh, C. M., Castillo Ramírez, L. A., and Ryu, S. (2013). Optogenetic elevation of endogenous glucocorticoid level in larval zebrafish. Front. Neural Circuits 7:82. doi: 10.3389/fncir.2013.00082

de Pedro, N., Alonso-Gómez, A. L., Gancedo, B., Valenciano, A. I., Delgado, M. J., and Alonso-Bedate, M. (1997). Effect of alpha-helical-CRF[9-41] on feeding in goldfish: involvement of cortisol and catecholamines. Behav. Neurosci. 111, 398403. doi: 10.1037//0735-7044.111.2.398

Engeszer, R. E., Patterson, L. B., Rao, A. A., and Parichy, D. M. (2007). Zebrafish in the wild: a review of natural history and new notes from the field. Zebrafish 4, 21-40. doi: 10.1089/zeb.2006.9997

Friedrich, R. W., Jacobson, G. A., and Zhu, P. (2010). Circuit neuroscience in zebrafish. Curr. Biol. 20, R371-R381. doi: 10.1016/j.cub.2010.02.039

Furuse, M., Matsumoto, M., Saito, N., Sugahara, K., and Hasegawa, S. (1997). The central corticotropin-releasing factor and glucagon-like peptide- 1 in food intake of the neonatal chick. Eur. J. Pharmacol. 339, 211-214. doi: 10.1016/s00142999(97)01391-5

Gahtan, E., and Baier, H. (2004). Of lasers, mutants and see-through brains: functional neuroanatomy in zebrafish. J. Neurobiol. 59, 147-161. doi: 10.1002/neu. 20000

Gao, Q., and Horvath, T. L. (2007). Neurobiology of feeding and energy expenditure. Annu. Rev. Neurosci. 30, 367-398. doi: 10.1146/annurev.neuro.30.051606. 094324

Glennemeier, K. A., and Denver, R. J. (2002a). Developmental changes in interrenal responsiveness in anuran amphibians. Integr. Comp. Biol. 42, 565-573. doi: 10. $1093 / \mathrm{icb} / 42.3 .565$

Glennemeier, K. A., and Denver, R. J. (2002b). Role for corticoids in mediating the response of Rana pipiens tadpoles to intraspecific competition. J. Exp. Zool. 292, 32-40. doi: 10.1002/jez.1140

Gregory, T. R., and Wood, C. M. (1999). The effects of chronic plasma cortisol elevation on the feeding behaviour, growth, competitive ability and swimming performance of juvenile rainbow trout. Physiol. Biochem. Zool. 72, 286-295. doi: $10.1086 / 316673$

Herget, U., Wolf, A., Wullimann, M. F., and Ryu, S. (2014). Molecular neuroanatomy and chemoarchitecture of the neurosecretory preoptic-hypothalamic area in zebrafish larvae. J. Comp. Neurol. 522, 1542-1564. doi: 10.1002/cne. 23480

Janzen, W. J., Duncan, C. A., and Riley, L. G. (2012). Cortisol treatment reduces ghrelin signaling and food intake in tilapia, Oreochromis mossambicus. Domest. Anim. Endocrinol. 43, 251-259. doi: 10.1016/j.domaniend.2012. 04.003

Kennedy, G. C. (1953). The role of depot fat in the hypothalamic control of food intake in the rat. Proc. R. Soc. Lond. B Biol. Sci. 140, 578-596. doi: 10.1098/rspb. 1953.0009

Kimmel, C. B., Ballard, W. W., Kimmel, S. R., Ullmann, B., and Schilling, T. F. (1995). Stages of embryonic development of the zebrafish. Dev. Dyn. 203, $253-$ 310. doi: 10.1002/aja.1002030302
Kimmel, C. B., Patterson, J., and Kimmel, R. O. (1974). The development and behavioral characteristics of the startle response in the zebra fish. Dev. Psychobiol. 7, 47-60. doi: 10.1002/dev.420070109

Kohashi, T., Nakata, N., and Oda, Y. (2012). Effective sensory modality activating an escape triggering neuron switches during early development in zebrafish. J. Neurosci. 32, 5810-5820. doi: 10.1523/JNEUROSCI.6169-11. 2012

Löhr, H., and Hammerschmidt, M. (2011). Zebrafish in endocrine systems: recent advances and implications for human disease. Annu. Rev. Physiol. 73, 183-211. doi: 10.1146/annurev-physiol-012110-142320

Lowry, C. A., and Moore, F. L. (2006). Regulation of behavioral responses by corticotropin-releasing factor. Gen. Comp. Endocrinol. 146, 19-27. doi: 10. 1016/j.ygcen.2005.12.006

MacPhail, R. C., Brooks, J., Hunter, D. L., Padnos, B., Irons, T. D., and Padilla, S. (2009). Locomotion in larval zebrafish: influence of time of day, lighting and ethanol. Neurotoxicology 30, 52-58. doi: 10.1016/j.neuro.2008. 09.011

Maniam, J., and Morris, M. J. (2012). The link between stress and feeding behaviour. Neuropharmacology 63, 97-110. doi: 10.1016/j.neuropharm.2012. 04.017

Matsuda, K., Azuma, M., Maruyama, K., and Shioda, S. (2013). Neuroendocrine control of feeding behavior and psychomotor activity by pituitary adenylate cyclase-activating polypeptide (PACAP) in vertebrates. Obes. Res. Clin. Pract. 7, e1-e7. doi: 10.1016/j.orcp.2012.10.002

McElligott, M. B., and O'malley, D. M. (2005). Prey tracking by Larval Zebrafish: axial kinematics and visual control. Brain Behav. Evol. 66, 177-196. doi: 10. $1159 / 000087158$

McFarland, D. (1971). Feedback Mechanisms in Animal Behaviour. London, New York: Academic Press.

McHenry, M. J., Feitl, K. E., Strother, J. A., and Van Trump, W. J. (2009). Larval zebrafish rapidly sense the water flow of a predator's strike. Biol. Lett. 5, 477479. doi: 10.1098/rsbl.2009.0048

Merali, Z., Graitson, S., Mackay, J. C., and Kent, P. (2013). Stress and eating: a dual role for bombesin-like peptides. Front. Neurosci. 7:193. doi: 10.3389/fnins.2013. 00193

Morton, G. J., Meek, T. H., and Schwartz, M. W. (2014). Neurobiology of food intake in health and disease. Nat. Rev. Neurosci. 15, 367-378. doi: 10. 1038/nrn3745

Nguyen, M., Yang, E., Neelkantan, N., Mikhaylova, A., Arnold, R., Poudel, M. K., et al. (2013). Developing 'integrative' zebrafish models of behavioral and metabolic disorders. Behav. Brain Res. 256, 172-187. doi: 10.1016/j.bbr.2013. 08.012

Olszewski, J., Haehnel, M., Taguchi, M., and Liao, J. C. (2012). Zebrafish larvae exhibit rheotaxis and can escape a continuous suction source using their lateral line. PLoS One 7:e36661. doi: 10.1371/journal.pone.0036661

Ortega, V. A., Lovejoy, D. A., and Bernier, N. J. (2013). Appetite-suppressing effects and interactions of centrally administered corticotropin-releasing factor, urotensin I and serotonin in rainbow trout (Oncorhynchus mykiss). Front. Neurosci. 7:196. doi: 10.3389/fnins.2013.00196

Oswald, M., and Robison, B. D. (2008). Strain-specific alteration of zebrafish feeding behavior in response to aversive stimuli. Can. J. Zool. 86, 1085-1094. doi: 10.1139/z08-085

Øverli, Ø., Winberg, S., Damsård, B., and Jobling, M. (1998). Food intake and spontaneous swimming activity in Arctic char (Salvelinus alpinus): role of brain serotonergic activity and social interactions. Can. J. Zool. 76, 1366-1370. doi: 10. 1139/z98-050

Pankhurst, N. W., Ludke, S. L., King, H. R., and Peter, R. E. (2008). The relationship between acute stress, food intake, endocrine status and life history stage in juvenile farmed Atlantic salmon, Salmo salar. Aquaculture 275, 311-318. doi: 10. 1016/j.aquaculture.2008.01.001

Portugues, R., Severi, K. E., Wyart, C., and Ahrens, M. B. (2013). Optogenetics in a transparent animal: circuit function in the larval zebrafish. Curr. Opin. Neurobiol. 23, 119-126. doi: 10.1016/j.conb.2012.11.001

Roberts, A. C., Reichl, J., Song, M. Y., Dearinger, A. D., Moridzadeh, N., Lu, E. D., et al. (2011). Habituation of the C-start response in larval zebrafish exhibits several distinct phases and sensitivity to NMDA receptor blockade. PLOS one 6:e29132. doi: 10.1371/journal.pone.0029132

Sapolsky, R. M. (2000). Stress hormones: good and bad. Neurobiol. Dis. 7, 540-542. doi: $10.1006 /$ nbdi. 2000.0350 
Selye, H. (1950). Stress and the general adaptation syndrome. Br. Med. J. 1, 13831392. doi: 10.1136/bmj.1.4667.1383

Selye, H. (1956). Endocrine reactions during stress. Curr. Res. Anesth. Analg. 35, 182-193. doi: 10.1213/00000539-195605000-00004

Spence, R., Gerlach, G., Lawrence, C., and Smith, C. (2008). The behaviour and ecology of the zebrafish, Danio rerio. Biol. Rev. Camb. Philos. Soc. 83, 13-34. doi: 10.1111/j.1469-185x.2007.00030.x

Spina, M., Merlo-Pich, E., Chan, R. K., Basso, A. M., Rivier, J., Vale, W., et al. (1996). Appetite-suppressing effects of urocortin, a CRF-related neuropeptide. Science 273, 1561-1564. doi: 10.1126/science.273.5281.1561

Steenbergen, P. J., Richardson, M. K., and Champagne, D. L. (2011). The use of the zebrafish model in stress research. Prog. Neuropsychopharmacol. Biol. Psychiatry 35, 1432-1451. doi: 10.1016/j.pnpbp.2010.10.010

Stewart, W. J., Cardenas, G. S., and McHenry, M. J. (2013). Zebrafish larvae evade predators by sensing water flow. J. Exp. Biol. 216, 388-398. doi: 10.1242/jeb. 072751

Teichner, W. H. (1968). Interaction of behavioral and physiological stress reactions. Psychol. Rev. 75, 271-291. doi: 10.1037/h0020281

Tinbergen, N. (1951). The Study of Instinct. Oxford: Clarendon Press.

Trivedi, C. A., and Bollmann, J. H. (2013). Visually driven chaining of elementary swim patterns into a goal-directed motor sequence: a virtual reality study of zebrafish prey capture. Front. Neural Circuits 7:86. doi: 10.3389/fncir.2013. 00086

Upton, K. R., and Riley, L. G. (2013). Acute stress inhibits food intake and alters ghrelin signaling in the brain of tilapia (Oreochromis mossambicus). Domest. Anim. Endocrinol. 44, 157-164. doi: 10.1016/j.domaniend.2012. 10.001
Wada, H., and Breuner, C. W. (2008). Transient elevation of corticosterone alters begging behavior and growth of white-crowned sparrow nestlings. J. Exp. Biol. 211, 1696-1703. doi: 10.1242/jeb.009191

Wendelaar Bonga, S. E. (1997). The stress response in fish. Physiol. Rev. 77, 591-625.

Westerfield, M. (2000). The Zebrafish Book. A Guide for the Laboratory use of Zebrafish (Danio Rerio). Eugene: University of Oregon Press.

Yeh, C. M., Glock, M., and Ryu, S. (2013). An optimized whole-body cortisol quantification method for assessing stress levels in larval zebrafish. PLoS One 8:e79406. doi: 10.1371/journal.pone.0079406

Zeddies, D. G., and Fay, R. R. (2005). Development of the acoustically evoked behavioral response in zebrafish to pure tones. J. Exp. Biol. 208, 1363-1372. doi: $10.1242 /$ jeb.01534

Conflict of Interest Statement: International patent No. WO2014/086938 describes some of the concepts presented in this manuscript.

Received: 11 July 2014; accepted: 05 October 2014; published online: 20 October 2014. Citation: De Marco RJ, Groneberg AH, Yeh C-M, Treviño M and Ryu S (2014) The behavior of larval zebrafish reveals stressor-mediated anorexia during early vertebrate development. Front. Behav. Neurosci. 8:367. doi: 10.3389/fnbeh.2014.00367 This article was submitted to the journal Frontiers in Behavioral Neuroscience. Copyright (C) 2014 De Marco, Groneberg, Yeh, Treviño and Ryu. This is an open-access article distributed under the terms of the Creative Commons Attribution License (CC $B Y)$. The use, distribution and reproduction in other forums is permitted, provided the original author(s) or licensor are credited and that the original publication in this journal is cited, in accordance with accepted academic practice. No use, distribution or reproduction is permitted which does not comply with these terms. 